\title{
Bacteriocins from Neisseria gonorrhoeae and their possible role in epidemiological studies
}

\author{
J. FLYNN AND M. G. MCENTEGART \\ From the Department of Medical Microbiology, The University of Sheffield, Sheffield
}

SYNOPSIS Although no consistent results can be demonstrated when freshly isolated strains of Neisseria gonorrhoeae are tested for bacteriocin activity on chocolate blood agar, such activity can be demonstrated on GC base medium (Difco), enriched with a defined supplement. At the present time, using six indicator strains, $75 \%$ of isolates of $N$. gonorrhoeae show characteristic patterns of inhibition. These observations are encouraging and suggest that 'gonocin' typing may be possible.

Despite the prevalence of the disease, no really satisfactory typing method for gonococci has yet been devised, although Hutchinson (1970) reported some success using a serological method based on the Lancefield technique. This lack of any means of identifying 'strains' of gonococci is a major handicap in the epidemiology of the disease. In an attempt to make good this defect, freshly isolated strains of gonococci were tested for their ability to produce bacteriocins. Bacteriocin-like activity against other fresh isolates was found to be produced by $75 \%$ of gonococci tested. It now seems likely that a sufficient number of characteristic gonocin patterns may be observed to be of epidemiological interest.

\section{Materials and Methods}

All strains were isolated from patients attending two local VD clinics. Primary cultures were made on chocolate blood agar containing $5 \mu \mathrm{g} / \mathrm{ml}$ of vancomycin. After inoculation, the plates were incubated for 18 hours at $37^{\circ} \mathrm{C}$ in $10 \% \mathrm{CO}_{2}$ and positive identification was made on the basis of Gram stain, oxidase test, and fermentation of sugars.

The method finally adopted to test for bacteriocin production was a simple modification of that described by Abbott and Shannon (1958) for shigellae, and Gillies and Govan (1966) for Pseudomonas pyocyanea. Two media-Columbia chocolate blood agar and Difco G.C. base medium plus $2 \%$ defined supplement (White and Kellog, 1965)-were used initially. Soon, as it became obvious that reactions on chocolate blood agar were weak and inconsistent, its use was discontinued.

Received for publication 9 June 1971.
Inocula from pure cultures of freshly isolated strains were seeded as a heavy band about $1 \mathrm{~cm}$ wide across the centre of the medium in a glass petri dish. After 24 hours' incubation at $37^{\circ} \mathrm{C}$ the growth was removed with a glass slide and the surface of the plate treated with chloroform vapour for 15 minutes. Traces of chloroform were allowed to evaporate by exposing the plate to the air for a further 30 minutes. As the gonocins are stable, the plates at this stage can be stored at $4^{\circ} \mathrm{C}$ and when a number of plates are available for testing, the indicator strains can be applied to them all in a single convenient session.

Strains of gonococci to act as indicators were selected initially by trial and error, cross streaking each member of a group of isolates against all the others and selecting suitable ones for further testing. In order to prevent the degradation of the selected strains by repeated subculture, they were suspended in $10 \%$ horse serum broth and frozen in liquid nitrogen. At the same time a number of samples of each strain were freeze dried. When strains from liquid nitrogen were required for use they were thawed rapidly and grown on chocolate agar. The growth was scraped off and a suspension made in a small volume in nutrient broth. The indicator strains were then streaked across the original inoculum at right angles, using a platinum loop of $3 \mathrm{~mm}$ in diameter. The plates were then incubated in $10 \%$ $\mathrm{CO}_{2}$ at $37^{\circ} \mathrm{C}$ for 18 hours and the results of inhibition of indicator strains were recorded as either complete inhibition, thinning of growth only, or no inhibition. The patterns of inhibition are at present based on a series of six indicator strains which showed distinctive reactions. It is not suggested that six 
indicator strains is an adequate number, nor that the ones we have so far tested are the best. Nevertheless, using the method to examine 100 recently isolated strains of Neisseria gonorrhoeae, $75 \%$ were found to be 'typable'. The patterns obtained were quite consistent in that the same strains gave the same patterns on repeated typing.

It has not been possible to study the nature of the bacteriocin so far, as the classical methods of induction, eg, exposure to ultraviolet light or addition of mitomycin $\mathrm{C}$ to liquid cultures, have failed to induce gonocin production. Indeed, the growth of gonococci is inhibited in liquid culture by concentrations of mitonycin as low as $0.5 \mu \mathrm{g} / \mathrm{ml}$.

\section{Results}

One hundred isolates have been examined by this technique; $75 \%$ were found to be 'typable' and so far 13 patterns of inhibition have been found to occur using the limited number of indicator strains at present available.

\section{Discussion}

The use of gonocins as a method of typing Neisseria gonorrhoeae appears to be possible and could prove valuable, especially in the absence of any other satisfactory method. Similar observations on Neisseria meningitidis were made by Kingsbury (1966) when he demonstrated that strains which were identical serologically could be placed in distinct bacteriocin groups.
Unlike the typing of Gram-negative bacilli, the bacteriocin typing of gonococci poses the additional problem of the inherent instability of cultures, especially those being used as indicators. Any method of gonocin typing must take into account the effect of changes in colony types of stock cultures.

Although the present method shows some $25 \%$ to be 'untypable', we feel that this will be reduced as more suitable indicator strains are seleced.

Further work is in progress to standardize the conditions of the test and to assess its value as an epidemiological tool.

This work was supported by a grant from the Medical Research Council.

We are grateful to Dr R. S. Morton and to colleagues at the Public Health Laboratory, Northern General Hospital, for strains of gonococci, and also to Miss Anne Littlewood for invaluable technical help.

\section{References}

Abbott, J. D., and Shannon, R. (1958). A method for typing Shigella sonnei, using Colicine production as a marker. J. clin. Path., 11, 71-77.

Gillies, R. R., and Govan, J. R. W. (1966). Typing of Pseudomenas pyocyanea by pyocine production. J. Path. Bact., 91, 339-345.

Hutchinson, I. R. (1970). Typing the gonococcus. (Letter). Brit. med. J., 3, 107.

Kingsbury, D. T. (1966). Bacteriocin production by strains of Neisseria meningitidis. J. Bact., 91, 1696-1699.

White, L. A., and Kellogg, D. S., Jr. (1965). Neisseria gonorrhoeae identification in direct smears by a fluorescent antibodycounterstain method. Appl. Microbiol., 13, 171-174. 